\title{
Bioelectrics in Basic Science and Medicine: Impact of Electric Fields on Cellular Structures and Functions
}

Stephen J. Beebe*

Frank Reidy Research Center for Bioelectrics, Old Dominion University, Norfolk, VA, USA

\begin{abstract}
Bioelectrics is a new interdisciplinary field that investigates electric field effects on cell membranes and other cellular components. It incorporates four main technologies, including electroporation, nanosecond pulsed electric fields, picosecond pulsed electric fields and cold plasmas. The parent technology in Bioelectrics is electroporation, which uses milli- and/or micro-second electric pulses to permeabilize cells and tissues, for delivery of membrane impermeable molecules. It is now being used for electro-gene delivery, with vascular endothelial growth factor, for revascularization in wound healing and cardiovascular and peripheral vascular disease. Plasmids expressing IL-12 are being delivered for immune system activation in melanoma treatment, now in phase II clinical trials. DNA vaccine delivery by electroporation is also being investigated. More recently, electroporation has been extended to include nanosecond pulsed electric fields (nsPEFs), a pulse power technology that was originally designed for military applications. It stores intense levels of electric energy, and then unleashes nanosecond bursts of instantaneous power into cells and tissues, creating unique intracellular conditions of high power and low, non-thermal energy. It is presently being used for cancer ablation of skin and internal tumors, and for platelet activation for wound healing in injury and diabetes. An extension of nsPEFs is to make the pulses even shorter, using picosecond pulsed electric fields. This is being developed as an imaging system to detect cancer and other aberrant tissues, using an antenna. The fourth technology is cold plasmas or ionized gasses, a fourth state of matter. Applications of these ionized gases are being developed for decontaminating wounds, water, food and surfaces. Other possible applications that are of specific interest, but not yet fully investigated, and/or developed, are pain control, fat ablation and decontamination of indwelling catheters. This review will outline some applications of Bioelectrics, with greatest focus on nsPEF effects on cells in vitro and tumors in vivo.
\end{abstract}

Keywords: Electroporation; Nanosecond pulsed electric fields; Cancer ablation; Apoptosis

\section{A New Interdisciplinary Field In Bioelectrics}

Bioelectrics is a burgeoning new interdisciplinary field, unifying an interdisciplinary team of scientists who integrate knowledge of electrical principles and theory, modeling and simulations, physics, material sciences, molecular cell biology, animal sciences and medicine. It investigates interactions of electric fields with cells and tissues for a number of possible basic science and therapeutic applications. There are also possible environmental applications. Bioelectrics was initially realized, but not specifically defined or named, when it was found that microsecond electric field effects on plasma membranes enhanced the penetration of DNA into mouse L-cells developing stable transformants [1]. This electric field mediated DNA transfer was called electroporation, and a model was proposed that electric field interactions with lipid dipoles in a pore configuration enhanced transport across the plasma membrane. More recently, pulse power technology, which originally was used for military purposes, has been applied to cells and tissues. This has led to some new and innovative possibilities for a number of potential uses. Of specific interest is to determine the nature of electric field interactions with cell membranes and other cellular structures, to determine how these effects alter cell functions, and to determine how Bioelectrics can be applied for basic science and medical advantages.

\section{Applications of bioelectrics}

Ageneral overview of uses for Bioelectrics includes applications that deliver DNA to cells and tissues, transiently or permanently alter cell membranes and other cell constituents, modulate cell signaling mechanisms, disinfect and decontaminate. Therapeutic applications include treating cancer [2-7], coronary and peripheral vascular disease [8]; activating platelets to enhance wound healing [9]; modulating cardiomyocyte action potentials [10]; transfecting plasmids for protein expression to improve wound healing [11], and to enhance immune responses [12]; vaccinating against diseases [13]; possibly imaging malignancies [14], and controlling pain by blocking nerve conduction [15]. Other applications include using electric fields [16,17] and cold plasmas [18], for sterilizing liquids, foods and surfaces; purifying air and water [19], and removing oxides of nitrogen and sulfur from diesel exhaust [20]. Another new field emerging in Bioelectrics is plasma medicine, where room temperature atmospheric pressure plasmas are used for sterilization, hygiene and dental/medical applications [21].

In this review, a major focus will be on applying milli-, microand nano-second pulsed electric fields (nsPEFs) in basic science and medicine. Most space will be filled on efficacy and mechanisms of cancer ablation with nsPEFs, in vitro and in vivo. For topics of materials sciences and engineering, the emphasis is to analyze interactions of electric field waveforms with biological materials, including cell membranes and other cellular components for medical applications.

*Corresponding author: Stephen J. Beebe, Frank Reidy Research Center for Bioelectrics, Old Dominion University, Norfolk, VA, USA, E-mail: SBeebe@odu.edu

Received December 19, 2012; Accepted February 15, 2013; Published February 18,2013

Citation: Beebe SJ (2013) Bioelectrics in Basic Science and Medicine: Impact of Electric Fields on Cellular Structures and Functions. J Nanomed Nanotechol 4 163. doi: $10.4172 / 2157-7439.1000163$

Copyright: ( 2013 Beebe SJ. This is an open-access article distributed under the terms of the Creative Commons Attribution License, which permits unrestricted use, distribution, and reproduction in any medium, provided the original author and source are credited. 


\section{Electroporation for Drug and Gene Delivery}

As indicated above, the most notable and recognized application of Bioelectrics is the use of external electric fields on biological cells to permeabilize cell membranes by electroporation [1]. In practice, electroporation is used to deliver impermeable molecules, such as nucleic acids, drugs or other molecules obstructed by plasma membranes. With potential problems for DNA delivery by viruses, delivery of plasmids or other nucleic acids to cells and tissues by electroporation has been shown to be safe and effective in humans [12]. It was also shown that only minor histological changes occurred in electroporated muscle [22], with neither little or no changes in gene profiles for major tumor suppressor genes or oncogenes of the cell cycle, nor change in expression of genes involved in DNA stability [23]. One practical medical application for electroporation is to permeabilize tumor cell membranes for the delivery of poorly permeable drugs, such as bleomycin, in a procedure called electrochemotherapy (ECT). Although not generally used in the US, ECT is now widely and successfully used in Europe for the treatment of cancer [24]

\section{Electrogene therapy for cancer treatment}

Use of the C57Bl/6 mouse harboring B16F10 melanoma tumors demonstrated feasibility for therapeutic applications of electro-gene therapy (EGT) to treat tumors. Initial thoughts were to use EGT for replacing defective genes. However, a major focus has been on several basic strategies for cancer therapy, including immune potentiation, suicide gene therapy, restoration of tumor suppressor genes, inhibition of oncogenes, anti-angiogenic gene therapy, delivering genes encoding toxins, or siRNAs to knockdown proteins important for survival and growth [25-27]. The delivery of a plasmid for IL-12 to metastatic melanoma tumors showed safety, efficacy reproducibility and titratability in Phase I clinical trials [12]. 42\% of melanoma patients who had no other approved treatment options showed disease stabilization or partial responses. In addition, 2 of 19 patients with non-electroporated distant lesions and no other systemic therapy showed complete regression of all metastases. Phase II clinical trials are ongoing. In pre-clinical trials [28], EGT utilizing a plasmid encoding for vascular endothelial growth factor (VEGF) demonstrated that wound healing could be accelerated [11]. Other studies found that high antibody titers after electroporation delivery of a plasmid encoding Hepatitis B surface antigen could be achieved after two applications [13].

\section{Electrogene therapy for cardiovascular disease}

EGT has also been shown to be effective in vivo to ischemic swine hearts, as a potential therapeutic approach for treatment of heart diseases [8]. Using three different penetrating electrodes and one non-penetrating electrode, the authors have shown that gene transfer to ischemic hearts via electroporation can be a safe and effective, non-viral method for delivering genes in vivo. Eight electric pulses of various widths and field strengths were synchronized to the rising phase of $\mathrm{R}$ waves of electrocardiograms, following an injection of either a plasmid encoding luciferase, or one encoding green fluorescent protein. Four different sites on the anterior wall of the left ventricle were treated. Expression was significantly higher in all electroporated sites, when compared to sites with plasmid injection only. It will now be interesting to determine if therapeutic genes such as VEGF can be effectively delivered to hearts after myocardial infarction, and if the expressed gene can improve cardiac function following an ischemic event.
Cell survival and DNA delivery during electroporation of course EGT requires that cells survive electroporation treatment, so they can express the gene of interest. Thus, there is a threshold transmembrane voltage that establishes permeabilization, another threshold that optiwmizes gene delivery and minimizes cell death; however, this condition may or may not be the condition that optimizes gene expression. Finally, there is a threshold that completely compromises cell viability, such that cells are irreversibly permeabilized [1,29-31]. Using this technique of Irreversible Electroporation (IRE) [32], is now in clinical trials for liver cancer treatment.

Exactly how electric fields affect cell membranes and other cellular mechanisms is still not completely understood. The term electroporation implies that "pores" or "aqueous channels" are formed in plasma membranes. This is consistent with transport of ions across membranes, as determined by a number of methods, including molecular dynamic simulations [33,34] and patch clamp studies [35], among others. However, it is unlikely that DNA diffuses into cells through these so-called pores. There is general agreement that the primary role in pore formation is played by water dipoles as they create water defects, and as they increasingly interact with electric fields at the lipid-water interface; initial steps in pore formation do not depend on the nature of lipid headgroups [33,34]. A number of steps are required for DNA entry into cells: electrophoretic migration of DNA towards the cell, DNA insertion into the membrane, translocation across the membrane, migration of DNA towards the nucleus, and finally transfer of DNA across the nuclear envelope [29,35-37]. Continuing investigations now suggest that these electric fields affect endocytotic mechanisms, engulfing DNA in membrane bound vesicles [38-40]. While this may account for how DNA gets into cells, it remains to be determined how DNA is released from these vesicles, enters the nucleus, and is engaged for gene transcription.

\section{Pulse Power Technology And Nanosecond Pulsed Electric Fields (nsPEFs)}

\section{Comprehending pulse power}

All electric methods discussed above use millisecond or microsecond pulses and relatively low electric fields. For ECT and EGT, electric field strengths are in the range of $\mathrm{V} / \mathrm{cm}$; however, IRE electric fields are generally in the low $\mathrm{kV} / \mathrm{cm}$ range. A more recent approach is to make greater use of pulse power technology by shortening pulse durations into the nanosecond domain, and increasing electric field strengths into the range of tens or hundreds of $\mathrm{kV} / \mathrm{cm}$ [41-43]. By storing electric energy in capacitors and releasing it in sub-microsecond bursts, electric fields can be delivered to cells and tissues with unique qualities of high peak power and low energy. When pulse repetition rates are relatively low, effects are non-thermal. The power is high because it is released in nanosecond durations. An example of pulse power principles is to compare storage of one joule of energy released in one second versus releasing the same energy in one microsecond or one nanosecond. If the stored joule of energy is released all at once in one second, the peak power delivered would only be 1 watt. If all of the stored energy were released within one microsecond, the power would be one megawatt, a million times greater; if released in one nanosecond, the peak power would be one gigawatt, a billion times greater. This is enough power to light a medium sized city for that nanosecond.

\section{Perceiving the nanosecond time domain}

To conceptualize nanoseconds, consider that the blink of an eye takes about 0.2 seconds or 200 milliseconds (ms). So, 60 ms would be 
about one quarter of the blink of an eye, or a very quick blink. Then, 60 nanoseconds would be $1 / 4000$ of the blink of an eye. Another comparison is to consider the distance that light travels in a given amount of time. Light travels 300 million meters/sec, or about 0.3 meters/ns, or about 1 foot. For pulse durations used in studies discussed here, often 60-100 ns, light would travel about 20-30 yards; the distance for 2 or 3 first downs on a football field. Of specific interests here are the consequences of these nanosecond bursts, and the interactions of these intense electric fields with cell and tissue structures, and ultimately, with cell and tissue functions.

\section{Intracellular effects and nanoporation with nsPEFs}

Unlike conventional electroporation pulses, nanosecond pulses are hypothesized to affect intracellular membranes. Therefore, a major focus has been on nsPEF effects on the endoplasmic reticulum, other calcium stores, mitochondria, and/or nuclei. The premise states that pulses with short durations and rapid (short) pulse rise and fall times charge intracellular membranes, which would have different effects on cell structures, and functions compared to conventional electroporation. When considering this pulse rise-fall principle in the frequency domain instead of the time domain, these rise-fall times would exhibit transient high frequency components, which affect intracellular membranes [44,45]. The initial concept of nsPEF effects was that plasma membranes were not affected or less affected. In experiments with cells in vitro, calcein did not escape from intracellular vesicles into the cytoplasm [41], and propidium iodide (PI) did not initially enter cells $[42,46]$, suggesting that plasma membranes remained intact. Later it became clear that breaches in plasma membranes were too small to allow escape or admission of molecules with sizes larger than about a nanometer, slighter smaller than calcein and PI. Modeling results showed nsPEF-induced conditions of supra-electroporation or formation of high density nanopores in all cell membranes $[47,48]$, which were later demonstrated experimentally. Experimental results demonstrated that cell membranes were depolarized and ions smaller than calcein and PI, such as TI [49] and $\mathrm{Ca}^{2+}$ [45] crossed plasma membranes under certain conditions, without transport of these larger molecules. Plasma membranes were depolarized and membrane potentials approached zero after cells were exposed to nsPEFs [50]. When nsPEFs were shown to eliminate cancer cells, evidence was interpreted as markers for apoptosis [2,51,52] and necrosis [50]. Such mechanisms could generally result from effects on plasma membranes, intracellular membranes, or both.

\section{In Vitro Evidence For Cell Death Mechanisms In Response To nsPEFs}

\section{Extrinsic or intrinsic cell death?}

It was hypothesized that nsPEF-induced apoptosis resulted from supra-electroporation [48]. Again, this could be due to extrinsic effects on plasma membranes, or intrinsic effects on endoplasmic reticulum, mitochondria and/or nuclei/DNA. Lysosomes could also be affecting, but have not been analyzed. In vitro studies in B16f10 melanoma [53] and E4 squamous cell carcinoma (SCC) [54] suggested that extrinsic apoptosis was at least partly involved, and caspase-independent cell death mechanisms were also implicated. For activation of an extrinsic pathway, it was possible that nsPEF effects on plasma membranes would aggregate Fas receptors. This would induce formation of the death-induced signaling complex (DISC), which is composed of Fas associated death domain (FADD), and inactive caspase- 8 binding to the intracellular domains of the Fas receptor. By this mechanism, caspase- 8 would be activated. Caspase- 8 could then directly activate caspase-3 (type I cells) or cleave Bid (type II cells), which would lead to cytochrome $c$ release, activation of caspase- 9 , and then, activation of caspase- 3 .

\section{Extrinsic cell death?}

To test for extrinsic apoptotic cell death, wild type Jurkat clones were compared to clones deficient in FADD $(\triangle \mathrm{FADD})$ or caspase- 8 $(\Delta$ caspase-8) [55]. Caspase activities $[-8,-9,-3]$ were increased in electric field- and time-dependent manners in all clones. When these clones were treated with ten $60 \mathrm{~ns}$ pulses with increasing electric fields $(0-60 \mathrm{kV} / \mathrm{cm})$, there were no differences in electric field effects on cell viability. All of these clones had electric field LD 50 values of about $30 \mathrm{kV} / \mathrm{cm}$. However, it has been shown that nearly all cell types will die when given lethal apoptotic stimuli, in the presence of caspase inhibition with the pan caspase inhibitor z-VAD-fmk [56,57]. Thus, in $\triangle F A D D$ and $\Delta$ caspase- 8 caspase experiments, this did not really rule out an extrinsic apoptosis pathway in nsPEF treated cells. However, it did show that like other apoptosis stimuli, nsPEFs activate similar caspase-independent cell death mechanisms in the absence of caspase activities.

To more specifically test for nsPEF-activated extrinsic apoptosis, cells were analyzed for cytochrome $\mathrm{c}$ release in the presence of the pan caspase inhibitor $\mathrm{z}$-VAD-fmk. Under these conditions, if the extrinsic pathway were activated, cytochrome $c$ release would be decreased in nsPEF-stimulated cells. However, results revealed that cytochrome c release was not affected by lethal nsPEF conditions at times up to 6 hours after treatment [55]. This indicated that the extrinsic pathway, and/or other pathways that may require FADD or caspase-8 were not functional in Jurkat cells, in response to nsPEFs.

\section{Intrinsic cell death}

Having ruled out the extrinsic pathway, the intrinsic pathway was tested using a Jurkat clone deficient in APAF-1 ( $\triangle \mathrm{APAF}-1)$ [55]. APAF1 is required in the intrinsic pathway to form the apoptosome, including a complex of APAF-1 with cytochrome c, d-ATP and caspase-9, which activates caspase- 9 . Caspase- 9 , then, activates caspase-3. It was shown that nsPEFs did not activate caspase- 3 in $\triangle \mathrm{APAF}-1$, but did in the vector control and wild type clone [55]. When analyzing cell viability, the vector control exhibited a linear electric-field dependent decrease in viability for $0-60 \mathrm{kV} / \mathrm{cm}$, with significant decreases in cell viability at $20 \mathrm{kV} / \mathrm{cm}$. In contrast, $\triangle \mathrm{APAF}-1$ did not show significant decreases in cell viability, until about $40 \mathrm{kV} / \mathrm{cm}$. Between 50 and $60 \mathrm{kV} /$ $\mathrm{cm}$, there were no differences in cell viability between the two clones. These results demonstrated for the first time that nsPEF-induced cell death was APAF-1- and caspase-dependent at lower electric fields, but caspase-independent at higher electric fields. Thus, in Jurkat cells, nsPEF-induced cell death occurred through intrinsic pathways in the functional absence of extrinsic apoptosis involving FADD and caspase- 8

\section{NsPEF subcellular targets for cell death induction}

Although type I and type II extrinsic pathways were not operative in response to nsPEFs, roles for plasma membranes, intracellular membranes, mitochondria and DNA/nuclei in cell death had not been fully investigated. Release of calcium from the endoplasmic reticulum (ER) previously had been shown as an intracellular effect of nsPEFs $[42,58]$. In addition, nsPEFs were shown to dissipate the mitochondria membrane potential $(\Delta \Psi \mathrm{m})[53,54,59,60]$. To carry these analyses further, it was of interest to determine how increases 
Citation: Beebe SJ (2013) Bioelectrics in Basic Science and Medicine: Impact of Electric Fields on Cellular Structures and Functions. J Nanomed Nanotechol 4: 163. doi:10.4172/2157-7439.1000163

in intracellular calcium and loss of $\Delta \Psi \mathrm{m}$ were involved in cell death. N1-S1 hepatocellular carcinoma (HCC) cells we used [44,45], which are used in an in vivo orthotopic HCC model in rats. Calcium release from intracellular stores was not observed in N1-S1 cells, using Fluo4 as the calcium indicator. Since release of calcium from the ER had been shown before $[42,58]$, this suggested that calcium was not released from intracellular stores in N1-S1 HCC cells, that Fluo-4 was not sufficiently sensitive to detect it, or that increases in calcium were transient and absent when they were analyzed in these studies 10-30 minutes after treatment. However, nsPEF-induced influx of calcium through plasma membranes was readily evident, as was dissipation of $(\Delta \Psi \mathrm{m})$. To determine roles for intracellular calcium and $\Delta \Psi \mathrm{m}$ on cell viability, the theory was directly tested that high frequency components of nsPEFs (transient pulse features), which are determined by rapid (short) rise and fall times of pulses, are important for maximizing electric field interactions with intracellular membranes [44,45]. Here, the intracellular effect was dissipation of $\Delta \Psi \mathrm{m}$. Using one $600 \mathrm{~ns}$ pulse with fast (15 ns) and slow (150 ns) rise-fall times and increasing electric fields, pulses with short rise-fall times had greater effects on dissipation of $\Delta \Psi \mathrm{m}$, confirming the theory that transient pulse features of nsPEFs were an important component for intracellular effects. In addition, decreases in viability were parallel with dissipation of $\Delta \Psi \mathrm{m}$. Moreover, under conditions of a slow rise-fall time and a mismatched load, influx of $\mathrm{Ca}^{2+}$ was observed, but loss of $\Delta \Psi \mathrm{m}$ and cell viability were absent [45]. These findings indicated that loss in cell viability was determined by dissipation of $\Delta \Psi \mathrm{m}$. However, effects on $\Delta \Psi \mathrm{m}$ were not readily observed in the absence of calcium, indicating that nsPEFinduced dissipation of $\Delta \Psi \mathrm{m}$ was calcium-dependent. Given that most, if not all, calcium-regulated responses are mediated by proteins, a possible explanation for this finding is that effects of nsPEFs may be on the mitochondria permeability transition pore, which is voltageand calcium-dependent [61]. This suggestion requires additional experimentation.

\section{A Model And Overview Of Subcellular Targets And Cell Death Pathways Activated By nsPEFs In Vitro}

Figure 1 shows a scheme of our present understanding of possible subcellular targets and cell death mechanisms in response to nsPEFs, and summarizes the finding presented above. The model is mostly derived from experiments in human Jurkat cells. The dark green elements and arrows show primary pathways and subcellular effects, and the light green elements and arrows show secondary events that are involved, as cells die in response to nsPEFs. The muted elements do not appear to be operative in this model. Five possible primary targets were considered for nsPEF-induced cell death. These included aggregation of Fas receptors in plasma membranes [1], effects [2] on plasma membrane poration, on intracellular $\mathrm{Ca}^{2+}$ stores $[3,4]$ on mitochondria, or on nuclei [5], and/or DNA.

\section{Mitochondria and plasma membranes are nsPEF targets}

Based on the evidence presented above and elsewhere [44,45,55], mitochondria and the $\Delta \Psi \mathrm{m}$ appear to be primary targets for nsPEFs cell death was correlated with loss of $\Delta \Psi \mathrm{m}$. With one nsPEF waveform, cells responded with an influx of $\mathrm{Ca}^{2+}$, but without loss of $\Delta \Psi \mathrm{m}$ and cell viability. However, dissipation of $\Delta \Psi \mathrm{m}$ did not occur in the absence of calcium influx through plasma membranes. Thus, plasma membranes were also targets for nsPEFs. The presence of nanopores in the plasma membrane was observed when influx of $\mathrm{Ca}^{2+}$ could be seen at lower

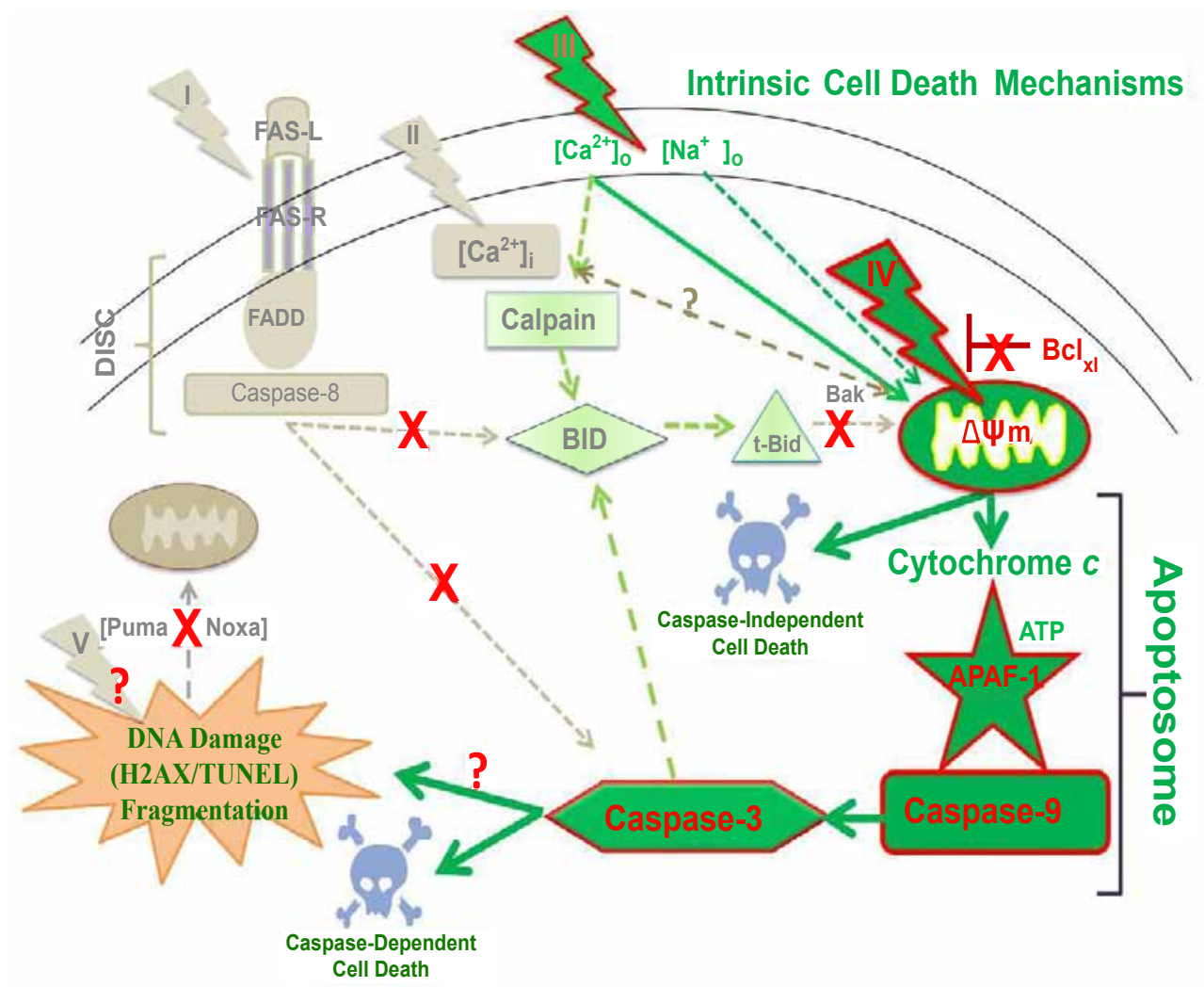

Figure 1: A model of cell death targets and mechanisms of nsPEF-induced cell death in Jurkat cells. 
electric fields than influx of propidium iodide [44]. These data fit the "two hit" hypothesis that requires a toxic stimulus, the nsPEF, as well as the presence of high $\mathrm{Ca}^{2+}$, as two requirements for opening the mPTP and disruption of $\Delta \Psi \mathrm{m}[62,63]$. Effects of nsPEFs on intracellular $\mathrm{Ca}^{2+}$ stores or on influx of $\mathrm{Ca}^{2+}$ alone were not sufficient for cell death in N1-S1 cells, because release of intracellular $\mathrm{Ca}^{2+}$ or $\mathrm{Ca}^{2+}$ influx without effects on $\Delta \Psi \mathrm{m}$ did not lead to cell death [45].

\section{Activation of the apoptosome}

Thus, nsPEFs cause an influx of $\mathrm{Ca}^{2+}$, a decrease in $\Delta \Psi \mathrm{m}$, especially with fast rise-fall times, release of cytochrome $c[51,55]$ and activation of caspase- 9 and -3 [55]. This occurs through formation of the apoptosome, clearly indicating activation of well-characterized, intrinsic mitochondria-, APAF-1-mediated apoptosis pathway. In addition, at high electric fields, nsPEF induced caspase-independent cell death. Both caspase-dependent and -independent pathways most likely originate due to effects on mitochondria in cells exhibiting different fundamental cellular contexts.

\section{Bid cleavage}

In these studies, caspases appear to cleave Bid, but this does not lead to cytochrome $c$ release through the type II extrinsic pathway [55]. This must happen downstream of caspase- 3 activation. The influx of $\mathrm{Ca}^{2+}$ most likely also leads to activation of calpain [54], which cleaves Bid, yet this does not seem to play a role in cytochrome $c$ release because cytochrome $c$ release is not affected by calpain inhibitors [45]. It is anticipated that the impact of nsPEFs on mitochondria is sufficient intense to exert maximum influence on cytochrome $c$ release, leaving little of no effect on $\mathrm{t}$-Bid on that event.

\section{DNA damage}

Although DNA damage by double strand breaks was present in this Jurkat cell model as determined by Histone 2AX phosphorylation, its role in cell death has not yet been clearly determined. NsPEF-induced DNA damage has been demonstrated in a number of cell types by a number of assays in vitro $[2,4,64,65]$, and in vivo $[5,6]$. In the Jurkat model here, the presence of DNA double strand breaks did not induce increases in Puma or Noxa, suggesting DNA damage was not a major mechanism to activate intrinsic apoptosis in response to nsPEF [45]. While such damage could lead to cell death, cell death has not been shown to be DNA damage-dependent. This is in contrast to cell death that has been shown to be dependent on $\mathrm{Ca}^{2+}$-influx, on dissipation of $\Delta \Psi \mathrm{m}$, or on caspase activation shown here.

\section{The Fas receptor is not involved}

Aggregation or other possible mechanisms for activating Fas receptors or the DISC do not appear to play a role in nsPEF-induced cell death; cytochrome $c$ release was not affected by inhibition of caspases with z-VAD-fmk [55], which would be required if cell death signals were initiated through formation of the DISC.

\section{What causes the loss of $\Delta \Psi \mathrm{m}$ ?}

Effects of nsPEFs have generally been considered to occur on membranes, so the most likely possibility for dissipation of $\Delta \Psi \mathrm{m}$ is nanoporation of the inner mitochondria membrane, as suggested by the supra-electroporation theorem [47,48]. Nevertheless, poration of the plasma membrane is necessary, and the requirement for $\mathrm{Ca}^{2+}$ for dissipation of $\Delta \Psi \mathrm{m}$ appears to come from the extracellular environment. However, poration is not expected to be $\mathrm{Ca}^{2+}$-dependent. Under these considerations, the most likely event is an effect of
nsPEFs on a calcium- and/or voltage-dependent mechanism(s) of the mitochondria permeability transition pore (mPTP) [61]. The rationale for this conjecture is based on the requirement for $\mathrm{Ca}^{2+}$ for loss of $\Delta \Psi \mathrm{m}$, and the evidence that most if not all effects of $\mathrm{Ca}^{2+}$ are mediated through actions on proteins. The supposition of nsPEF effects on proteins is unexpected and requires further study.

\section{In Vivo Evidence For Mechanisms Of Cell Death And Successful Ablation Of Tumors With nsPEFs}

\section{NsPEFs and cancer hallmarks}

In tumor tissues, nsPEFs were first shown to reduce mouse B10.2 squamous cell carcinoma tumor size and induce apoptosis as shown by TUNEL and activation of caspase catalytic activity $[2,66]$. They also have been shown to eliminate mouse ectopic B16f10 tumors [35], ectopic mouse Hepa1-6 hepatocellular carcinoma [6], and murine xenographs of human pancreatic carcinoma [67]. It was of specific interest to determine mechanisms for tumor eradication based on rate limiting-stochastic events involved with cancer growth and treatment. A major complication in treating cancer is that it is really hundreds of different diseases; even melanoma or liver cancer is often not a single disease, not only among patients, but likely within the same patient. For example, the recurrence of a treated cancer is often a different cancer, even in the same organ [68]. Thus, cancers exhibit hundreds of different genotypes defined by substantial numbers of mutations in a wide variety of genes/proteins. In order to manage this array of diseases and to provide more focused characterization of cancer, Hanahan and Weinberg $[69,70]$ defined eight major hallmarks of cancer that exhibit physiological anomalies that control cell homeostasis and proliferation. These include self-sufficiency in growth signals, insensitivity to growth-inhibitory (antigrowth) signals, evasion of apoptosis, limitless replicative potential, sustained angiogenesis, tissue invasion and metastasis, reprogramming energy metabolism and evasion of immune surveillance. In addition, genetic diversity of cancers is caused by an unstable genome. Further, inflammation advances multiple cancer hallmarks [70]. Considering these cancer hallmarks, it was relevant to determine which of them were altered by nsPEFs. In two different cancers, it has been shown that nsPEFs impinge upon multiple hallmarks, and can thereby, mimic several different specific treatments. For example, angiostatin and endostatin inhibit sustained angiogenesis; vinblastine and vincristine affect limitless replicative potential.

\section{Activation of caspase-associated and -unassociated cell death by nsPEFs}

In both B16f10 [5] and Hepa1-6 tumors [6], nsPEFs were shown to induce apoptosis, as shown by activation of caspases, well-defined indicators of apoptosis. The time course for activation of caspases-2, -6 and -7 mirrored cell shrinkage, decreases in nuclear size and nuclear condensation. These characteristics were paralleled by DNA damage, as indicated by TUNEL and histone 2AX phosphorylation; the latter is specific for DNA double strand breaks. The coincidence of these makers suggests that they were all part of the causes, and/or results of nsPEF effects on cancer cells. However, these studies also showed that not all cells exhibited active caspases after nsPEF treatment. This is consistent with the in vitro E4 SCC [54] and Jurkat cell [55] studies mentioned above, demonstrating both caspase-dependent and -independent cell death.

\section{Anti-angiogenesis/anti vascular effects with nsPEFs}

Other evidence suggested that nsPEFs reversed sustained 
angiogenesis [5,6]. First, there was a decrease in vessel numbers supplying tumors that was coincident with a decrease in tumor mass, indicating an interruption of tumor-driven angiogenesis and tumor blood supply. It could also be argued that there is an anti-vascular effect. This was also shown by Doppler analysis of blood flow in murine B16f10 melanoma [3]. Second, over a three week period after treatment of nsPEFs, vascular endothelial growth factor (VEGF) progressively decreased such that VEGF levels were one sixth the pre-treatment levels $[5,6]$. VEGF is a requirement for the angiogenic switch that defines homeostasis. This results in a loss of balance between stimulation and inhibition of new blood vessel growth. The angiogenic switch defines an early step for cancer metastasis and multistage carcinogenesis $[69,70]$. There were also decreases in platelet-derived endothelial cell growth factor, a well-known chemotactic factor for vascular endothelial cells and blood vessel formation. Moreover, effectors downstream of VEGF were decreased, including three major microvascular density markers: CD31, a platelet-endothelial cell adhesion molecule, used as a panendothelial cell marker; CD34, an endothelial cell marker and CD105, a proliferation-related endothelial cell marker. Thus, while melanoma and hepatocellular carcinoma tumors shrank and vessel numbers were significantly reduced, formation of new vessels was extensively inhibited.

\section{NsPEFs and immune surveillance}

In research that is ongoing and in need of further study, nsPEFs may reverse evasion of immune surveillance in nsPEF treated mouse ectopic Hepa1-6 HCC [71], and orthotopic N1-S1 HCC. In 6 of 8 tumors that were cleared of Hepa1-6 HCC tumors for 60 days, none of the six grew new tumors for 49 days after being challenged with another tumor cell injection in the opposite flank. In contrast, all 8 age-matched naïve mice readily grew tumors. Similar results have also been observed in a UV-induced murine melanoma study [72]. After treatment with nsPEFs, challenge tumor cell injections resulted in slower growth of tumors cleared by nsPEFs than in mice treated by surgically removing tumors. This suggests that after treatment, the immune system might have alerted to recognize primary tumor cells as foreign. Thus, nsPEF treatment may induce immunogenic cell death, which has been shown to occur more readily when cells die in response to some, but not all apoptotic stimuli $[73,74]$. This suggests that successful treatment with nsPEFs may induce a vaccine response in the treated host that would prevent recurrence of tumors, which is a major problem in cancer therapy.

\section{Advantages of nsPEF Ablation For Cancer Treatment}

So what advantages do nsPEFs provide over other cancer treatments? First, nsPEFs target multiple cell death mechanisms. In in vitro experiments with B16f10 melanoma, E4 SSC and Jurkat cell models, as well as the ectopic models of melanoma and HCC, more than one mechanism for cell death was present. Second, nsPEFs produce a well-defined treatment zone, which is defined by a center active needle and four surrounding ground electrode in a 5 needle array [75]. When all tumor cells are placed within these treatment zone boundaries and are exposed to electric field strength, above an ablation threshold for a sufficient time, all tumor cells will die by more than one mechanism. Third, as shown in Jurkat cells and N1-S1 HCC models in vitro, nsPEF appears to target mitochondria, and the $\Delta \Psi \mathrm{m}$, especially when the plasma membrane is permeabilized and calcium is present. Fourth, nsPEFs can bypass cancer mutations when tumor cells are exposed to an ablation electric field for an appropriate period of time. Tumor cells that evade apoptosis by expressing mutation affecting the Fas and
DISC pathways, or otherwise interfere with caspase activation will still die by caspase-independent mechanisms. Fifth, in addition to the antiangiogenesis effects of nsPEFs, they also have another advantage by local infarction of small vessels, as shown by the decrease in blood vessel numbers in the treatment zone [5]. Sixth, it can be argued that this treatment also has an anti-vascular effect within the treatment zone. A seventh advantage is the absence of local or systemic side effects by this treatment. While the treatment does involve delivery of electric fields, unpublished clinical trials indicated that injection of a local anesthetic, such as lidocaine, nullifies any pain. Eighth, since pulses are so short, there is for the most part, no muscle contraction as was shown when normal pig liver was treated with nsPEFs [75]. However, when treating ectopic tumors, it is important to prevent the electrode from coming intact with muscle or nerves leading to them, and to have the animal properly grounded during the treatment. Ninth, when electric fields are sufficiently intense, all cells in the treatment zone can be killed, including cancer stem cells and host cells that provide needed growth factors for tumor growth and metastasis. Finally, confirmation of the enhanced immune surveillance in humans after nsPEFs could provide a highly significant advantage to nsPEF treatment of tumors.

\section{Future Directions With nsPEF Treatment Of Cancer}

Pre-clinical studies indicate the feasibility and general safety for using nsPEF treatments for several types of cancer. While early studies were carried out with a mouse melanoma model $[3,4,72]$, this is not the best disease model for clinical application for cancer treatment because melanoma is a systemic and metastatic disease, while nsPEF ablation is a localized treatment. More applicable cancers include squamous cell carcinoma [54,76] and basal cell carcinoma [7,77]. These are generally not metastatic, and are readily accessible and treatable malignancies for nsPEFs.

We are presently finishing a series of studies using an orthotopic rat N1-S1 HCC model that shows excellent results. Our future goals are to treat larger HCC tumors with shorter treatment times. Also, we have specific interests in ongoing research in the orthotopic model for possible positive effects of nsPEFs, to enhance immune surveillance after treatment. We are in pursuit to identify mechanisms that are responsible for the absence of growth of a second challenge tumor cell injection, after successful nsPEF treatment of the primary tumor [71]. Other efforts are to develop applications of nsPEFs to include minimally invasive, laparoscopic treatments with catheter electrodes, guided by ultrasound using the rabbit VX2 HCC tumor model.

Because cancers are always treated with more than one therapy, either in combination or in sequence, we have considered using low non-toxic doses of chemotherapeutic agents, before or after treatment with nsPEFs. We have recently demonstrated that nsPEFs and gemcitabine act synergistically to effectively treat an oral squamous cell carcinoma in vitro [78]. This would allow treatments of larger tumors with lower electric fields.

We continue to be interested in mechanisms of nsPEF-induced cell death, and the N1-S1 tumor cells exhibits some behaviors that are different that previously observed. These tumor cells present a paradox. We see relative high caspase activity in untreated cells in vitro [79], that deceases with time and with increasing electric fields. However, in vivo, we see active caspase- 3 in populations of cells in tissue slices from treated tumors. Nevertheless, as reported here and elsewhere $[44,45]$, the nsPEF target is a calcium-dependent decrease in $\triangle \Psi \mathrm{m}$ in these cells. 
Citation: Beebe SJ (2013) Bioelectrics in Basic Science and Medicine: Impact of Electric Fields on Cellular Structures and Functions. J Nanomed Nanotechol 4: 163. doi:10.4172/2157-7439.1000163

\section{The State Of And The Future For Bioelectrics}

There are growing numbers of investigators in Bioelectric Centers around the World that are investigating all aspects of Bioelectrics, included at the beginning of this review. For example, the Frank Reidy Research Center for Bioelectrics at Old Dominion University now includes 17 principle investigators, occupying about 28,000 square feet in a new building that includes new state of the art animal facility and state of the art diagnostic and investigational equipment and instruments. There is now a Consortium for Bioelectrics, including 3 Centers in the US, 2 in France, 2 in Germany, and 1 each in Italy, Slovenia and Japan. There are also other groups who are not part of the Consortium working on Bioelectrics in the US, Germany, France, Korea, Singapore, Japan and China. It is highly likely that the number of investigators will continue to grow, and new applications will be realized as Bioelectrics research continues to evolve.

\section{Acknowledgements}

The author thanks $\mathrm{Dr}$.John $\mathrm{D}$. Robertson and his colleagues from the Departments of Pharmacology, Toxicology and Therapeutics, University of Kansas Cancer Center, Kansas City, Kansas, for generously providing the APAF1 deficient clone and its vector control [80]. We also thank Mr. Frank Reidy for his generous financial support of this project. We also appreciate funding for this project from the Commonwealth Research and Commercialization Fund, Virginia Center for Innovative Technology. We apologize to the authors of many excellent published papers in this field that were not included in this short review.

\section{References}

1. Neumann E, Schaefer-Ridder M, Wang Y, Hofschneider PH (1982) Gene transfer into mouse lyoma cells by electroporation in high electric fields. Embo J 1: 841-845.

2. Beebe SJ, Fox PM, Rec LJ, Somers K, Stark RH, et al. (2002) Nanosecond pulsed lectric field (nsPEF) effects on cells and tissues: apoptosis induction and tumor growth inhibition. IEEE Trans Plasma Sci 30: 286-292.

3. Nuccitelli R, Pliquett U, Chen X, Ford W, Swanson RJ, et al. (2006) Nanosecond pulsed electric fields cause melanomas to self-destruct. Biochem Biophys Res Commun 343: 351-360

4. Nuccitelli R, Chen X, Pakhomov AG, Baldwin WH, Sheikh S, et al. (2009) A new pulsed electric field therapy for melanoma disrupts the tumor's blood supply and causes complete remission without recurrence. Int J Cancer 125: 438-445.

5. Chen X, Kolb JF, Swanson RJ, Schoenbach KH, Beebe SJ (2010) Apoptosis initiation and angiogenesis inhibition: melanoma targets for nanosecond pulsed electric fields. Pigment Cell Melanoma Res 23: 554-563.

6. Chen X, Zhuang J, Kolb JF, Schoenbach KH, Beebe SJ (2012) Long term survival of mice with hepatocellular carcinoma after pulse power ablation with nanosecond pulsed electric fields. Technol Cancer Res Treat 11: 83-93.

7. Garon EB, Sawcer D, Vernier PT, Tang T, Sun Y, et al. (2007) In vitro and in vivo evaluation and a case report of intense nanosecond pulsed electric field as a local therapy for human malignancies. Int J Cancer 121: 675-682.

8. Hargrave B, Downey H, Strange R Jr, Murray L, Cinnamond C, et al. (2012) Electroporation-mediated gene transfer directly to the swine heart. Gene Ther 20: 151-157.

9. Zhang J, Blackmore PF, Hargrave BY, Xiao S, Beebe SJ, et al. (2008) Nanosecond pulse electric field (nanopulse): a novel non-ligand agonist for platelet activation. Arch Biochem Biophys 471: 240-248.

10. Wang S, Chen J, Chen MT, Vernier PT, Gundersen MA, et al. (2009) Cardiac myocyte excitation by ultrashort high-field pulses. Biophys J 96: 1640-1648.

11. Ferraro B, Cruz YL, Coppola D, Heller R (2009) Intradermal delivery of plasmid VEGF(165) by electroporation promotes wound healing. Mol Ther 17: 651-657.

12. Daud Al, DeConti RC, Andrews S, Urbas P, Riker Al, et al. (2008) Phase I trial of interleukin-12 plasmid electroporation in patients with metastatic melanoma. $\mathrm{J}$ Clin Oncol 26: 5896-5903.

13. Donate A, Coppola D, Cruz Y, Heller R (2011) Evaluation of a novel nonpenetrating electrode for use in DNA vaccination. PLoS One 6: e19181.

14. Kolb JF, Xiao S, Camp JT, Migliaccio M, Bajracharya C, et al. (2010) Sub- nanosecond electrical pulses for medical therapies and imaging. Proceedings of the Fourth European Conference on Antennas and Propagation (EuCAP) USA.

15. Joshi RP, Mishra A, Song J, Pakhomov A, Schoenbach KH (2008) Simulation studies of ultrashort, high-intensity electric pulse induced action potential block in whole-animal nerves. IEEE Trans Biomed Eng 55: 1391-1398.

16. Schoenbach KH, Katsuki S, Stark RH, Buescher ES, Beebe SJ (2002) Bioelectrics-new applications for pulsed power technology. IEEE Trans Plasma Sci 30: 293-300

17. Chalise PR, Perni S, Shama G, Novac BM, Smith R, et al. (2006) Lethality mechanisms in Escherichia coli induced by intense sub-microsecond electrical pulses. Appl Phys Lett 89: 153902.

18. Laroussi M (2005) Low Temperature Plasma-Based Sterilization: Overview and State-of-the-Art. Plasma Process Polym 2: 391-400.

19. Malik MA, Xiao S, Schoenbach KH (2012) Scaling of surface-plasma reactors with a significantly increased energy density for $\mathrm{NO}$ conversion. J Hazard Mater 209: 293-298.

20. Malik MA (2010) Water purification by plasmas: which reactors are most energy efficient? Plasma Chem Plasma Process 30: 21-31.

21. Kong MG, Kroesen G, Morfill G, Nosenko T, Shimizu T, et al. (2009) Plasma medicine: an introductory review. New J Phys 11: 115012.

22. Hojman P, Zibert JR, Gissel H, Eriksen J, Gehl J (2007) Gene expression profiles in skeletal muscle after gene electrotransfer. BMC Mol Biol 8: 56.

23. Mlakar V, Todorovic V, Cemazar M, Glavac D, Sersa G (2009) Electric pulse used in electrochemotherapy and electrogene therapy do not significantly change the expression profile of genes involved in the development of cancer in malignant melanoma cells. BMC Cancer 9: 299.

24. Miklavčič D, Serša G, Brecelj E, Gehl J, Soden D, et al. (2012) Electrochemotherapy: technological advancements for efficient electroporationbased treatment of internal tumors. Med Biol Eng Comput 50: 1213-1225.

25. Potter H, Heller R (2003) Transfection by Electroporation. Curr Protoc Mol Biol.

26. Heller LC, Heller R (2006) In vivo electroporation for gene therapy. Hum Gene Ther 17: 890-897.

27. Heller LC, Heller R (2010) Electroporation gene therapy preclinical and clinica trials for melanoma. Curr Gene Ther 10: 312-317.

28. Heller R, Shirley S, Guo S, Donate A, Heller L (2011) Electroporation based gene therapy--from the bench to the bedside. Conf Proc IEEE Eng Med Bio Soc 2011: 736-738.

29. Wolf H, Rols MP, Boldt E, Neumann E, Teissié J (1994) Control by pulse parameters of electric field-mediated gene transfer in mammalian cells. Biophys J 66: 524-531.

30. Rols MP, Teissié J (1998) Electropermeabilization of mammalian cells to macromolecules: control by pulse duration. Biophys J 75: 1415-1423.

31. Marjanovic I, Haberl S, Miklavcic D, Kanduser M, Pavlin M (2010) Analysis and comparison of electrical pulse parameters for gene electrotransfer of two different cell lines. J Membr Biol 236: 97-105.

32. Davalos RV, Mir IL, Rubinsky B (2005) Tissue ablation with irreversible electroporation. Ann Biomed Eng 33: 223-231.

33. Levine ZA, Vernier PT (2010) Life cycle of an electropore: field-dependent and field-independent steps in pore creation and annihilation. J Membr Biol 236 $27-36$

34. Tieleman DP (2004) The molecular basis of electroporation. BMC Biochem 5: 10.

35. Pakhomov AG, Bowman AM, Ibey BL, Andre FM, Pakhomova ON, et al. (2009) Lipid nanopores can form a stable, ion channel-like conduction pathway in cell membrane. Biochem Biophys Res Commun 385: 181-186.

36. Hristova NI, Tsoneva I, Neumann E (1997) Sphingosine-mediated electroporative DNA transfer through lipid bilayers. FEBS Lett 415: 81-86.

37. Favard C, Dean DS, Rols MP (2007) Electrotransfer as a non viral method of gene delivery. Curr Gene Ther 7: 67-77.

38. Rols MP, Femenia P, Teissie J (1995) Long-lived macropinocytosis takes place 
Citation: Beebe SJ (2013) Bioelectrics in Basic Science and Medicine: Impact of Electric Fields on Cellular Structures and Functions. J Nanomed Nanotechol 4: 163. doi:10.4172/2157-7439.1000163

in electropermeabilized mammalian cells. Biochem Biophys Res Commun 208: 26-35

39. Satkauskas S, Bureau MF, Mahfoudi A, Mir LM (2001) Slow accumulation of plasmid in muscle cells: supporting evidence for a mechanism of DNA uptake by receptor-mediated endocytosis. Mol Ther 4: 317-323.

40. Antov Y, Barbul A, Mantsur H, Korenstein R (2005) Electroendocytosis: exposure of cells to pulsed low electric fields enhances adsorption and uptake of macromolecules. Biophys J 88: 2206-2223.

41. Schoenbach KH, Beebe SJ, Buescher ES (2001) Intracellular effect of ultrashort electrical pulses. Bioelectromagnetics 22: 440-448.

42. Vernier PT, Sun Y, Marcu L, Salemi S, Craft CM, et al. (2003) Calcium bursts induced by nanosecond electric pulses. Biochem Biophys Res Commun 310: 286-295

43. Beebe SJ, Schoenbach KH (2005) Nanosecond pulsed electric fields: a new stimulus to activate intracellular signaling. J Biomed Biotechnol 2005: 297-300.

44. Beebe SJ, Chen YJ, Sain NM, Schoenbach KH, Xiao S (2012) Transient features in nanosecond pulsed electric fields differentially modulate mitochondria and viability. PLoS One 7: e51349.

45. Beebe SJ, Sain NM, Ren W (2013) Induction of cell death mechanisms and apoptosis by nanosecond pulsed electric fields (nsPEFs). Cells.

46. Deng J, Schoenbach KH, Buescher ES, Hair PS, Fox PM, et al. (2003) The effects of intense submicrosecond electrical pulses on cells. Biophys $\mathrm{J} 84$ 2709-2714.

47. Stewart DA, Gowrishankar TR, Weaver JC (2004) Transport lattice approach to describing electroporation: use of a local asymptotic model. IEEE Trans Plasma Sci 32: 1696-1708.

48. Gowrishankar TR, Esser AT, Vasilkoski Z, Smith KC, Weaver JC (2006) Microdosimetry for conventional and supra-electroporation in cells with organelles. Biochem Biophys Res Commun 341: 1266-1276.

49. Bowman AM, Nesin OM, Pakhomova ON, Pakhomov AG (2010) Analysis of plasma membrane integrity by fluorescent detection of $\mathrm{TI}(+)$ uptake. $\mathrm{J} \mathrm{Membr}$ Biol 236: 15-26.

50. Pakhomov AG, Shevin R, White JA, Kolb JF, Pakhomova ON, et al. (2007) Membrane permeabilization and cell damage by ultrashort electric field shocks. Arch Biochem Biophys 465: 109-118.

51. Beebe SJ, Fox PM, Rec LJ, Willis EL, Schoenbach KH (2003) Nanosecond, high-intensity pulsed electric fields induce apoptosis in human cells. Faseb $J$ 17: $1493-1495$

52. Vernier PT, Li A, Marcu L, Craft CM, Gundersen MA (2003) Ultrashort pulsed electric fields induce membrane phospholipid translocation and caspase activation: differential sensititivities of Jurkat T lymphoblasts and rat glioma C6 cells. IEEE Trans Dielectr Electr Insul 10: 795-809.

53. Ford WE, Ren W, Blackmore PF, Schoenbach KH, Beebe SJ (2010) Nanosecond pulsed electric fields stimulate apoptosis without release of pro-apoptotic factors from mitochondria in B16f10 melanoma. Arch Biochem Biophys 497: 82-89.

54. Ren W, Beebe SJ (2011) An apoptosis targeted stimulus with nanosecond pulsed electric fields (nsPEFs) in E4 squamous cell carcinoma. Apoptosis 16: 382-393.

55. Ren W, Sain NM, Beebe SJ (2012) Nanosecond pulsed electric fields (nsPEFs) activate intrinsic caspase-dependent and caspase-independent cell death in Jurkat cells. Biochem Biophys Res Commun 421: 808-812.

56. Lawrence CP, Chow SC (2005) FADD deficiency sensitises Jurkat T cells to TNF-alpha-dependent necrosis during activation-induced cell death. FEBS Lett 579: 6465-6472.

57. Vonarbourg C, Stolzenberg MC, Hölzelova E, Fischer A, Deist FL, et al. (2002) Differential sensitivity of Jurkat and primary $T$ cells to caspase-independent cell death triggered upon Fas stimulation. Eur J Immunol 32: 2376-2384.

58. White JA, Blackmore PF, Schoenbach KH, Beebe SJ (2004) Stimulation of capacitative calcium entry in HL-60 cells by nanosecond pulsed electric fields. J Biol Chem 279: 22964-22972.
59. Batista Napotnik T, Wu YH, Gundersen MA, Miklavčič D, Vernier PT (2012) Nanosecond electric pulses cause mitochondrial membrane permeabilization in Jurkat cells. Bioelectromagnetics 33: 257-264.

60. Vernier PT (2011) Mitochondrial membrane permeabilization with nanosecond electric pulses. Conf Proc IEEE Eng Med Biol Soc 2011: 743-745.

61. Halestrap AP, Pasdois $P$ (2009) The role of the mitochondrial permeability transition pore in heart disease. Biochim Biophys Acta 1787: 1402-1415

62. Jacobson J, Duchen MR (2002) Mitochondrial oxidative stress and cell death in astrocytes--requirement for stored $\mathrm{Ca}^{2+}$ and sustained opening of the permeability transition pore. J Cell Sci 115: 1175-1188.

63. Brookes PS, Yoon Y, Robotham JL, Anders MW, Sheu SS (2004) Calcium ATP, and ROS: a mitochondrial love-hate triangle. Am J Physiol Cell Physio 287: C817-C833.

64. Stacey M, Stickley J, Fox P, Statler V, Schoenbach K, et al. (2003) Differentia effects in cells exposed to ultra-short, high intensity electric fields: cell survival, DNA damage, and cell cycle analysis. Mutat Res 542: 65-75.

65. Stacey M, Fox P, Buescher S, Kolb J (2011) Nanosecond pulsed electric field induced cytoskeleton, nuclear membrane and telomere damage adversely impact cell survival. Bioelectrochemistry 82: 131-134.

66. Beebe SJ, White J, Blackmore PF, Deng Y, Somers K, et al. (2003) Diverse effects of nanosecond pulsed electric fields on cells and tissues. DNA Cell Bio 22: 785-796.

67. Nuccitelli R, Huynh J, Lui K, Wood R, Kreis M, et al. (2013) Nanoelectroablation of human pancreatic carcinoma in a murine xenograft model without recurrence. Int J Cancer 132: 1933-1939.

68. Amaravadi RK, Flaherty KT (2007) Targeted therapy for metastatic melanoma Clin Adv Hematol Oncol 5: 386-394.

69. Hanahan D, Weinberg RA (2000) The hallmarks of cancer. Cell 100: 57-70.

70. Hanahan D, Weinberg RA (2011) Hallmarks of cancer: the next generation Cell 144: 646-674.

71. Beebe SJ, Ford WE, Ren W, Chen X (2011) Pulse power ablation of melanoma with nanosecond pulsed electric fields. In: Treatment of Metastatic Melanoma, R. Morton (Ed.), In Tech Croatia 231-268.

72. Nuccitelli R, Tran K, Lui K, Huynh J, Athos B, et al. (2012) Non-therma nanoelectroablation of UV-induced murine melanomas stimulates an immune response. Pigment Cell Melanoma Res 25: 618-629.

73. Green DR, Ferguson T, Zitvogel L, Kroemer G (2009) Immunogenic and tolerogenic cell death. Nat Rev Immunol 9: 353-363.

74. Ferguson TA, Choi J, Green DR (2011) Armed response: how dying cells influence T-cell functions. Immunol Rev 241: 77-88.

75. Long G, Shires P, Plescia D, Beebe SJ, Kolb JF, et al. (2011) Targeted Tissue Ablation with nanosecond pulses. IEEE Trans Biomed Eng 58: 2161-2167.

76. Yin D, Yang WG, Weissberg J, Goff CB, Chen W, et al. (2012) Cutaneous papilloma and squamous cell carcinoma therapy utilizing nanosecond pulsed electric fields (nsPEF). PLoS One 7: e43891.

77. Nuccitelli R, Tran K, Athos B, Kreis M, Nuccitelli P, et al. (2012) Nanoelectroablation therapy for murine basal cell carcinoma. Biochem Biophys Res Commun 424: 446-450.

78. Wang J, Guo J, Wu S, Feng H, Sun S, et al. (2012) Synergistic effects of nanosecond pulsed electric fields combined with low concentration of gemcitabine on human oral squamous cell carcinoma in vitro. PLoS One 7: e43213.

79. Beebe SJ, Chen X, Liu JA, Schoenbach KH (2011) Nanosecond pulsed electric field ablation of hepatocellular carcinoma. Conf Proc IEEE Eng Med Biol Soc 2011: 6861-6865.

80. Shawgo ME, Shelton SN, Robertson JD (2008) Caspase-mediated Bak activation and cytochrome $\mathrm{c}$ release during intrinsic apoptotic cell death in Jurkat cells. J Biol Chem 283: 35532-35538. 